\title{
Development and Validation of a Japanese Version of the Emotion Regulation Questionnaire for Children and Adolescents
}

This article was published in the following Dove Press journal:

Neuropsychiatric Disease and Treatment

\author{
Hikari Namatame ${ }^{1,2}$ \\ Hiroko Fujisato ${ }^{2}$ \\ Masaya Ito ${ }^{2}$ \\ Yoko Sawamiya $\mathbb{D}^{\prime}$
}

'Faculty of Human Sciences, University of Tsukuba, Tsukuba City, Ibaraki, Japan;

${ }^{2}$ National Center for Cognitive Behavior Therapy and Research, National Center of Neurology and Psychiatry, Kodaira City, Tokyo, Japan
Correspondence: Hikari Namatame Graduate School of Comprehensive Human Sciences, University of Tsukuba, I-I-I Tennodai, Tsukuba 305-8572, Japan Tel +8I-29-853-5609

Email hikari.namatame@gmail.com
Purpose: We developed a Japanese version of the Emotion Regulation Questionnaire for Children and Adolescents (ERQ-CA) and examined its reliability and validity across three studies.

Patients and Methods: In Study 1, the Japanese version of ERQ-CA was developed and administered to 389 children aged 8-12 years. In Study 2, the questionnaire was administered to 1738 adolescents aged 12-18 years. In Study 3, utilizing a sample of 1300 children and adolescents, the test was administered twice over a period of four weeks in order to assess test-retest reliability.

Results: In Study 1, the Japanese version of ERQ-CA showed the same factor structure as the original version, along with good internal consistency reliability and acceptable construct validity. In Study 2, the questionnaire's factor structure, internal consistency reliability, and construct validity were again confirmed. Finally, in Study 3, measurement invariance was tested across distinct age groups $(8-11,12-15$, and 16-18 years), and the questionnaire had good test-retest reliability over a period of four weeks.

Conclusion: The Japanese version of the ERQ-CA had good reliability and validity.

Keywords: adolescents, children, emotion regulation, Japan, reliability validity

\section{Introduction}

The number of studies on emotion regulation in children and adolescents has dramatically increased over the past two decades, mainly due to the association between emotion regulation and symptoms of psychopathology in children and adolescents. ${ }^{1}$ For children, emotional dysregulation can lead to an array of social problems in schools, ${ }^{2}$ while emotion regulation is a central correlate of behavioral and emotional problems in adolescents. ${ }^{3}$

Among studies on emotion regulation, the process model of emotion regulation proposed by Gross ${ }^{4}$ has had a profound influence. This focuses on two principal strategies: cognitive reappraisal (CR), which refers to a cognitive change that occurs prior to the generation of an emotion and modifies the emotional impact of a situation; and expressive suppression (ES), which involves the inhibition of emotion-expression behavior after an emotional response has been generated. ${ }^{5} \mathrm{CR}$ is conducted early in the emotion-generative process and decreases the intensity of an emotion while having no impact on memory. Contrastingly, ES occurs later in the emotion-generative process and fails to decrease emotion intensity. ${ }^{6}$ 
To assess these two emotion strategies, Gross and $\mathrm{John}^{7}$ developed an emotion regulation scale: the Emotion Regulation Questionnaire (ERQ). Then, Gullone and $\mathrm{Taffe}^{8}$ created children and adolescents' version of the ERQ (ERQ-CA), in which item expressions were modified to make them easier for children and adolescents to understand. The ERQ-CA consists of 10 items: six evaluate CR ("I control my feelings about things by changing the way I think about them") and four evaluate ES (e.g., "I keep my feelings to myself").

The reliability and validity of the ERQ-CA were investigated by administering it to a population of children and adolescents aged 10-18 years. ${ }^{8}$ Consequently, good alpha coefficients and stability (intraclass correlation) coefficients were shown for both CR and ES over a 12-month period, indicating good reliability. ${ }^{8}$ Regarding validity, construct validity using confirmatory factor analysis (CFA) was investigated, and a two-factor model was a suitable fit. ${ }^{8}$ In addition, adequate convergent validity was shown regarding the correlation between the ERQ-CA and depression and the "Big Five" personality traits (i.e., neuroticism, extraversion, agreeableness, conscientiousness, and openness to experience). ${ }^{8}$ Assessing CR and ES using ERQ-CA was determined to be a means of predicting mental manifestation in children and adolescents. Specifically, CR was significantly negatively correlated with both depression and anxiety, while ES was significantly positively correlated with these. ${ }^{9}$ Therefore, the ERQ-CA is a key tool for understanding the psychopathology of children and adolescents.

Although a Japanese version of the ERQ has been developed, ${ }^{10}$ there is, as of yet, no Japanese version of the ERQ-CA. Emotion regulation is essential for Asian children and adolescents, and some studies have suggested that emotion regulation strategies differ between Western and Eastern societies. Suppression occurs more frequently, and is less associated with negative emotion in Asian cultures compared to Western ones. ${ }^{11}$ To compare Western and Eastern emotion regulation strategies, it is necessary to develop and examine the reliability and validity of Asian language version of ERQ-CA. Clarifications of psychometric properties of ERQ-CA amongst this population are needed for facilitating further research to understanding the nature of emotion regulation in this population. In the current study, we consequently developed a Japanese version of the ERQ-CA (ERQ-CA-J) and examined its reliability and validity. This paper describes three studies, each of which examined various dimensions of the psychometric properties of the ERQ-CA-J.

\section{Study I}

The purpose of Study 1 was to develop the ERQ-CA-J and investigate its factor structure, internal consistency reliability, and construct validity among children aged $8-12$ years.

\section{Methods}

\section{Participants and Procedures}

The institutional review board (IRB) at the University of Tsukuba approved this study's ethical and scientific protocols (approval number: 筑29-117). Participants were recruited from a primary school in Japan after obtaining informed consent from the school's principal. After obtaining passive parental consent, whereby parents could choose to exclude their child from the research, the children's verbal assent was obtained by the parents, as they asked their children to participate. Additionally, in order to ensure that all children were informed and chose whether to participate in the study, the teachers also informed the children about the study and verbally asked the children to participate. The children were informed that the survey would take approximately 15-20 mins to complete, that their participation was voluntary, that they were free to withdraw at any time without giving a reason, and that all information collected would be anonymous and kept strictly confidential.

Four hundred twelve children completed the survey in the classroom with the support of a teacher; in other words, if they could not understand a word's meaning, they could ask the teacher. The researcher was not in the room when the surveys were completed. Twenty-three participants were omitted because their submissions contained significant missing data (i.e., leaving $>20 \%$ of the items blank in at least one measure). This resulted in a sample of 389 participants (aged 8-12 years, $\mathrm{M}_{\text {age }}=$ 10.30 years $\mathrm{SD}_{\text {age }}=1.23$ years; 202 boys, 186 girls, and one who did not specify sex).

\section{Measures}

ERQ-CA-J: The original ERQ-CA ${ }^{8}$ comprises 10 items assessing the emotion-regulation strategies of $\mathrm{CR}$ (six items) and ES (four items). Items are rated using a fivepoint Likert-type response scale $(1=$ "strongly disagree," 2 = "disagree," 3 = "half-and-half," 4 = "agree," 5 = "strongly agree"). The original ERQ-CA possesses a two-factor 
structure (ES and CR), with a correlated error added between item 1 ("when I want to feel happier, I think about something else") and item 3 ("when I want to feel better [e.g., less sad, angry, or worried], I think about something else"). ${ }^{8}$ Regarding reliability, the alpha coefficients were previously calculated to be 0.83 for $\mathrm{CR}$ and 0.75 for $\mathrm{ES}^{8}$ In addition, previous work found the stability (intraclass correlation) coefficients over a 12-month period to be moderate for both $\mathrm{CR}$ and ES. For CR, the intraclass correlation coefficients ranged in size from 0.37 (for 13-15-year-olds) to 0.47 (for 16-18-year-olds) in earlier studies, while for ES, the coefficients ranged in size between 0.40 (for 10-12-year-olds) and 0.63 (for 16-18-year-olds). ${ }^{8}$ Finally, the correlation between the original ERQ-CA and depression and the Big Five personality traits showed adequate convergent validity in a previous study; specifically, the correlation between depression was -0.26 for CR and 0.37 for ES. ${ }^{8}$

The COnsensus-based Standards for the selection of health Measurement INstruments (COSMIN), ${ }^{12}$ which are guidelines for assessing the methodological quality of studies on measurement properties of self-reported outcomes, were applied in the development of the ERQ-CA-J. After obtaining permission from the original developer to create the ERQ-CA-J, two authors, Japanese clinical psychologists, independently translated the original scale from English into Japanese. Then, two additional clinical psychologists discussed the clarity, language expression, and conceptual equivalence of the combined forward-translated version. Next, two bilingual individuals, unaware of the goal of the present study, independently back-translated the scale from Japanese to English. The research team conducted an examination of the conceptual equivalence of the two translated versions with the original version and highlighted problematic expressions. This process was repeated several times. The original developer of the ERQ-CA then confirmed the conceptual equivalence of the original and back-translated versions.

Children's Emotional Regulation Scale: The Children's Emotional Regulation Scale ${ }^{13}$ is an 18-item measure of emotion regulation for children originally developed in Japan. The scale has a three-factor structure: emotion inhibition (seven items; e.g., "I often suppress my anger"), difficulties concerning emotion regulation (six items; e.g., "I tend to lose my temper"), and coping with emotion regulation (five items; e.g., "When I am scared, I try to be with someone else"). Each item is rated using a five-point scale $(1=$ "strongly disagree" to $5=$ "strongly agree").
Spence Children's Anxiety Scale: The Spence Children's Anxiety Scale (SCAS) ${ }^{14,15}$ is a 38 -item measure of anxiety symptoms designed for children and adolescents. The SCAS has a six-factor structure: separation anxiety disorder, social phobia, generalized anxiety disorder, panic attacks and agoraphobia, obsessive-compulsive disorder, and physical injury fears.

The original version of the $\operatorname{SCAS}^{14}$ was determined through CFA and exploratory factor analysis to support six factors, and in the model the first-order factors loaded significantly onto a single second-order actor, generalized anxiety disorder. For internal consistency, alpha coefficients were 0.82 (panic agoraphobia), 0.70 (separation anxiety), 0.70 (social phobia), 0.60 (physical injury fears), 0.73 (obsessive-compulsive disorder), and 0.73 (generalized anxiety). ${ }^{14}$ Further, performing test-retest reliability over a six-month period showed a correlation coefficient of 0.60 for total score. ${ }^{14}$ Thus, the convergent and discriminant validity was supported. ${ }^{14}$

The Japanese version of the SCAS ${ }^{15}$ reported sufficient reliability coefficients $(0.94$ and 0.92$)$ for the full-scale scores among children and adolescents aged 9-15 years. In addition, the scale had sufficient test-retest reliability over 2-4 weeks: $r=0.76$ for children and $r=0.86$ for adolescents $(p s<0.001)$. Each item was rated using a fourpoint scale ( $0=$ "never" to $3=$ "always") .

Depression Self-Rating Scale: The Depression Self-rating Scale (DSRS) ${ }^{16,17}$ is an 18-item measure of depressive symptoms for children. The DSRS has a single-factor structure. The test-retest reliability of the scale was 0.80 which was sufficient. ${ }^{16}$ Each item had a reliability coefficient of $0.65-0.95 .^{16}$ The split-half reliability was 0.86 , which implied satisfactory internal consistency. ${ }^{16}$ The original version of the DSRS had adequate face and factorial validity. ${ }^{16}$ For clinical validity, 155 children aged 8-14 years who were psychiatry outpatients were grouped into a depressed population and others. ${ }^{18}$ Children who scored 15 and over on the DSRS were significantly more likely to have a depressive diagnosis. ${ }^{18}$ The DSRS total score could predict as well as the global score of the history of depression and appearance of depression rated at interview by Child Psychiatrists. ${ }^{18}$

The Japanese version of the DSRS showed good testretest reliability. ${ }^{18}$ The Cronbach's $\alpha$ was 0.77 , which was sufficient. ${ }^{17}$ For validity, the Japanese version of DSRS was significantly correlated $(r=0.71)$ with the Japanese version of the Children's Depression Inventory $(\mathrm{CDI})^{17}$ 
and with teachers' evaluations. ${ }^{17}$ Each item is rated using a three-point scale $(0=$ "never" to $2=$ "always") .

\section{Statistical Analyses}

Statistical analyses were conducted by IBM SPSS Statistics 25.0 and Amos 25.0. To examine the structural validity, of Japanese version of the ERQ-CA, we randomly split the whole sample into two subsamples. Then, the first sample (subsample 1) was used for exploratory factor analysis (EFA), and the second sample (subsample 2) for CFA, respectively. For EFA, the number of factors was estimated through the visual scree test. ${ }^{19}$ Maximum likelihood estimations using promax rotation were performed to explore factor loadings. An item was retained if it had (a) an item-factor loading of at least 0.35 on a primary factor, (b) cross-loadings at least 0.10 difference between factor loadings as guidelines for meaningful factor loadings generally range from 0.30 to $0.40 .^{20}$ For CFA, the scale parameter was estimated using the maximum likelihood method rather than Mean- and Variance-adjusted Weighted Least Squares (WLSMV), as the data were not categorical but quantitative. ${ }^{21}$ The model fit was determined based on the recommendations of $\mathrm{Hu}$ and Bentler: ${ }^{22}$ using the Comparative Fit Index (CFI) and the root mean square error of approximation (RMSEA). Specifically, CFI values of $\geq 0.95$ and RMSEA values of $\leq 0.06$ suggest a good fit of the model to the data, whereas CFI values of $0.90-0.94$ and RMSEA values of $0.07-0.10$ suggest an acceptable fit.

For correlation analyses, we assumed that even if Pearson's correlation values were very weak, they could show significance because of the sample size. Therefore, we adopted the criteria applied by Cohen, ${ }^{23}$ in which Pearson correlation values of $r= \pm 0.50$ are considered strong, $r= \pm 0.30$ are considered moderate, and $r= \pm 0.10$ are considered weak. Regarding the correlation between ES and other validity measures, it was hypothesized that ES would show a strong positive relationship with emotion inhibition, a moderate positive relationship with difficulties concerning emotion regulation, a weak positive relationship with coping with emotion regulation, a moderate positive relationship with anxiety, and a weak to moderate relationship with depression. For CR, it was hypothesized that it would show a strong negative relationship with emotion inhibition, a moderate negative relationship with difficulties concerning emotion regulation, a weak positive correlation with coping with emotion regulation, a moderate negative relationship with anxiety, and a weak to moderate relationship with depression.

\section{Results and Discussion \\ Descriptive Statistics}

The means and standard deviations for the two emotionregulation strategies (ES and CR) are shown in Table 1. The means were almost the same as those in the original version; however, the standard deviation of CR was larger. ${ }^{8}$

\section{Structural Validity}

We conducted EFA on subsample 1, to examine the unique factorial structure for the Japanese sample. The visual scree test indicated two-factor structure same as the original version as most appropriate. Table 2 shows the rotated factor pattern matrix and extraction communalities. Two factors explained $52.76 \%$ of the variance of ERQ-CA scores. Factor loadings on the first factor (CR) ranged $0.42-0.81$ and the second factor (ES), 0.42-0.80. The two factors were correlated $(r=0.57)$. Thus, the EFA indicated the Japanese version of the ERQ-CA has a twofactor structure same as the original version.

We conducted CFA on subsample 2, to confirm the results provided by EFA. Using CFA, a two-factor model based on the findings of Gross and John ${ }^{7}$ showed excellent fit $(\mathrm{CFI}=0.954$, RMSEA $=0.057)$. Following the recommendations of Gullone and Taffe, ${ }^{8}$ a correlated error was added between item 1 ("when I want to feel happier, I think about something else") and item 3 ("when I want to feel better [e.g., less sad, angry, or worried], I think about something else"), which have similarly worded phrases, and this produced a better fit $(\mathrm{CFI}=0.959$, RMSEA $=0.055$, see Table 3). Standardized item-factor loadings for CR ranged from 0.50 to 0.68 and $\mathrm{ES}$ ranged from 0.52 to 0.78 (Table 4), which were considered sufficient. Therefore, the

Table I Means (and Standard Deviations) for the Two Emotion Regulation Strategies (ES and CR)

\begin{tabular}{|l|l|l|l|}
\hline \multicolumn{2}{|l|}{ Sample } & \multicolumn{2}{l|}{ Emotion Regulation Strategy } \\
\cline { 3 - 4 } \multicolumn{2}{|l|}{} & ES & CR \\
\hline Study I & Overall & II.83(3.59) & $19.5 I(5.47)$ \\
\hline Study 2 & Overall & II.5I (3.27) & $19.33(4.57)$ \\
& Age 12-15 years & II.22(3.35) & $18.75(4.74)$ \\
& Age 16-18 years & II.85(3.15) & $20.02(4.27)$ \\
\hline \multirow{2}{*}{ Study 3 } & Overall & II.70(3.0I) & $19.77(4.45)$ \\
& Age 8-II years & $12.47(3.26)$ & $20.40(4.92)$ \\
& Age 12-15 years & II.33 (2.9I) & $19.24(4.34)$ \\
& Age 16-18 years & II.94 (2.87) & $20.52(4.11)$ \\
\hline
\end{tabular}

Note: Means and standard deviations for Study 3 were obtained the first time. Abbreviations: CR, cognitive reappraisal; ES, expressive suppression. 
Table 2 Exploratory Factor Analysis Factor Pattern Matrix

\begin{tabular}{|c|c|c|c|c|c|c|}
\hline \multirow[t]{2}{*}{ Item } & \multicolumn{3}{|c|}{ Study I } & \multicolumn{3}{|c|}{ Study2 } \\
\hline & $\begin{array}{l}\text { FI: } \\
\text { CR }\end{array}$ & $\begin{array}{l}\text { F2: } \\
\text { ES }\end{array}$ & $h^{2}$ & $\begin{array}{l}\text { FI: } \\
\text { CR }\end{array}$ & $\begin{array}{l}\text { F2: } \\
\text { ES }\end{array}$ & $h^{2}$ \\
\hline I. When I want to feel happier, I think about something different. & 0.42 & 0.14 & 0.26 & 0.47 & 0.02 & 0.23 \\
\hline 3. When I want to feel less bad (e.g., sad, angry or worried), I think about something different. & 0.67 & -0.02 & 0.44 & 0.57 & -0.01 & 0.32 \\
\hline 5. When I am worried about something, I make myself think about it in a way that helps me feel better. & 0.66 & -0.01 & 0.42 & 0.64 & -0.03 & 39 \\
\hline 7. When I want to feel happier about something, I change the way I am thinking about it. & 0.51 & 0.15 & 0.36 & 0.67 & 0.02 & 46 \\
\hline 8. I control my feelings about things by changing the way I think about them. & 0.66 & 0.03 & 0.45 & 0.69 & 0.06 & 51 \\
\hline $\begin{array}{l}\text { 10. When I want to feel less bad (e.g., sad, angry, or worried) about something, I change the way } \\
\text { I am thinking about it. }\end{array}$ & 0.81 & -0.10 & 0.57 & 0.75 & -0.05 & 53 \\
\hline 2. I keep my feelings to myself. & -0.13 & 0.71 & 0.41 & -0.10 & 0.78 & 0.56 \\
\hline 4. When I am feeling happy, I am careful not to show it. & 0.11 & 0.49 & 0.31 & -0.02 & 0.52 & 0.26 \\
\hline 6. I control my feelings by not showing them. & 0.03 & 0.80 & 0.66 & 0.06 & 0.66 & 0.48 \\
\hline 9. When I am feeling bad (e.g., sad, angry, or worried), I am careful not to show it. & 0.18 & 0.42 & 0.30 & 0.12 & 0.54 & 0.37 \\
\hline
\end{tabular}

soundness of the ERQ-CA-J's two-factor structure was indicated, similar to that of Gullone and Taffe. ${ }^{8}$

\section{Internal Consistency Reliability}

Cronbach's alpha coefficient was 0.70 for the ES items $(0.71$ for boys; 0.69 for girls), and 0.80 for the CR items ( 0.79 for boys; 0.78 for girls). Note that the result of full data set was higher than that of boys and girls, as the data set included data for one sex non-specified individual. Thus, the ERQ-CA-J demonstrated acceptable-to-moderate internal consistency.

\section{Construct Validity}

We conducted correlation analyses for two ERQ-CA-J factors, with other validity scales; the subscales of

Table 3 Goodness of Fit for ERQ-CA-J

\begin{tabular}{|l|l|l|l|l|}
\hline \multicolumn{2}{|l|}{ Model } & CFI & RMSEA & df \\
\hline Study I & Overall & 0.959 & 0.055 & 33 \\
\hline Study 2 & Overall & 0.923 & 0.077 & 33 \\
& Age 12-15 years & 0.930 & 0.075 & 33 \\
& Age 16-18 years & 0.910 & 0.080 & 33 \\
\hline \multirow{2}{*}{ Study 3 } & Overall & 0.908 & 0.075 & 33 \\
& Age 8-11 years & 0.897 & 0.079 & 33 \\
& Age 12-15 years & 0.876 & 0.086 & 33 \\
& Age 16-18 years & 0.912 & 0.074 & 33 \\
& Configural invariance model & 0.889 & 0.047 & 99 \\
& Factor loading invariance & 0.890 & 0.044 & 115 \\
& model & & & \\
& Intercept invariance model & 0.863 & 0.045 & 135 \\
\hline
\end{tabular}

Note: The results shown in this table represent the model where a correlated error was added between Item I and Item 3.

Abbreviations: CFI, Comparative Fit Index; $d f$, degrees of freedom; RMSEA, Root Mean Square Error of Approximation.
Children's Emotional Regulation Scale (emotion inhibition, difficulties concerning emotion regulation and coping with emotion regulation), SCAS, and DSRS (Table 5). The correlations between the ES score and the other validity scales were as expected.

On the other hand, the correlations between the CR score and the other validity scales differed from our expectations. A moderate positive correlation was shown between emotion inhibition and CR score, while a weak positive association was shown between $\mathrm{CR}$ score and difficulties concerning emotion regulation, coping with emotion regulation, and SCAS. There was almost no relation between DSRS and CR score. A possible explanation for this is that the $\mathrm{CR}$ and ES may not have been clearly differentiated among the children. For Japanese children, emotion-inhibition-related items similar to CR have the same factors. ${ }^{13}$

\section{Study 2}

The purpose of Study 2 was to develop the ERQ-CA-J and investigate its factor structure, internal consistency, and construct validity among adolescents aged $12-18$ years.

\section{Methods}

\section{Participants and Procedures}

The institutional review board (IRB) at the University of Tsukuba approved this study's ethical and scientific protocols (approval number: 筑29-117). Participants were recruited from a middle school and three high schools in Japan. Written informed consent was acquired from both the school's principal and the participants after the study was described to them. Of the 1779 participants, 41 were omitted from analyses because of significant 
Table 4 Confirmatory Factor Analysis Standardized Item-Factor Loadings

\begin{tabular}{|c|c|c|c|c|c|c|c|c|}
\hline \multirow[t]{2}{*}{ Items } & \multicolumn{2}{|l|}{ Study I } & Study 2 & \multicolumn{2}{|c|}{ Study 3} & \multirow[b]{2}{*}{$\begin{array}{l}\text { Age } \\
8-I I\end{array}$} & \multirow[b]{2}{*}{$\begin{array}{l}\text { Age } \\
12-15\end{array}$} & \multirow[b]{2}{*}{$\begin{array}{l}\text { Age } \\
16-18\end{array}$} \\
\hline & Overall & Overall & $\begin{array}{l}\text { Age } \\
12-15\end{array}$ & $\begin{array}{l}\text { Age } \\
16-18\end{array}$ & Overall & & & \\
\hline \multicolumn{9}{|l|}{ Cognitive reappraisal } \\
\hline I. When I want to feel happier, I think about something different. & 0.49 & 0.42 & 0.44 & 0.37 & 0.45 & 0.51 & 0.41 & 0.45 \\
\hline $\begin{array}{l}\text { 3. When I want to feel less bad (eg, sad, angry or worried), } \\
\text { I think about something different. }\end{array}$ & 0.61 & 0.48 & 0.48 & 0.45 & 0.58 & 0.59 & 0.56 & 0.65 \\
\hline $\begin{array}{l}\text { 5. When I am worried about something, I make myself think } \\
\text { about it in a way that helps me feel better. }\end{array}$ & 0.59 & 0.50 & 0.52 & 0.46 & 0.52 & 0.46 & 0.53 & 0.54 \\
\hline $\begin{array}{l}\text { 7. When I want to feel happier about something, I change the } \\
\text { way I am thinking about it. }\end{array}$ & 0.65 & 0.70 & 0.72 & 0.66 & 0.61 & 0.62 & 0.60 & 0.58 \\
\hline $\begin{array}{l}\text { 8. I control my feelings about things by changing the way I think } \\
\text { about them. }\end{array}$ & 0.63 & 0.74 & 0.75 & 0.72 & 0.66 & 0.66 & 0.65 & 0.62 \\
\hline $\begin{array}{l}\text { 10. When I want to feel less bad (e.g., sad, angry, or worried) } \\
\text { about something, I change the way I am thinking about it. }\end{array}$ & 0.65 & 0.67 & 0.65 & 0.72 & 0.69 & 0.68 & 0.69 & 0.72 \\
\hline \multicolumn{9}{|l|}{ Emotion suppression } \\
\hline 2. I keep my feelings to myself. & 0.61 & 0.69 & 0.68 & 0.68 & 0.59 & 0.59 & 0.57 & 0.54 \\
\hline 4. When I am feeling happy, I am careful not to show it. & 0.52 & 0.51 & 0.50 & 0.50 & 0.48 & 0.45 & 0.50 & 0.49 \\
\hline 6. I control my feelings by not showing them. & 0.73 & 0.78 & 0.77 & 0.79 & 0.77 & 0.69 & 0.78 & 0.88 \\
\hline $\begin{array}{l}\text { 9. When I am feeling bad (eg, sad, angry, or worried), I am } \\
\text { careful not to show it. }\end{array}$ & 0.50 & 0.58 & 0.60 & 0.56 & 0.49 & 0.56 & 0.44 & 0.51 \\
\hline
\end{tabular}

Note: The results shown in this table represent the model where a correlated error was added between Item I and Item 3.

Table 5 Study I Correlations

\begin{tabular}{|c|c|c|c|c|c|c|}
\hline & & \multicolumn{3}{|c|}{ Children's Emotional Regulation Scale } & \multirow[t]{2}{*}{ SCAS } & \multirow[t]{2}{*}{ DSRS } \\
\hline & & Emotion Inhibition & $\begin{array}{l}\text { Difficulty in Emotion } \\
\text { Regulation }\end{array}$ & Coping with Emotion Regulation & & \\
\hline \multirow[t]{2}{*}{ Overall Sample } & ES & $0.6 I^{* *}$ & $0.32 * *$ & $0.15^{*}$ & $0.49 * *$ & $0.15^{* *}$ \\
\hline & CR & $0.48 * *$ & $0.31^{* *}$ & $0.27 * *$ & $0.37^{* *}$ & 0.01 \\
\hline \multirow[t]{2}{*}{ Girls } & ES & $0.62 * *$ & $0.20 *$ & -0.03 & $0.49 * *$ & $0.20 *$ \\
\hline & $C R$ & $0.39 * *$ & $0.22 * *$ & $0.21^{*}$ & $0.38^{* *}$ & 0.01 \\
\hline \multirow[t]{2}{*}{ Boys } & ES & $0.58 * *$ & $0.38 * *$ & $0.27^{* *}$ & $0.46^{* *}$ & 0.08 \\
\hline & CR & $0.49 * *$ & $0.29 * *$ & $0.23 * *$ & $0.27^{* *}$ & -0.06 \\
\hline
\end{tabular}

Notes: $*_{p}<0.05, * * p<0.01$.

Abbreviations: CR, cognitive reappraisal; DSRS, Depression Self-Rating Scale; ES, expressive suppression; SCAS, Spence Children's Anxiety Scale.

missing data (leaving $>20 \%$ of the items blank for at least one measure). This resulted in a sample of 1738 participants $\left(\mathrm{M}_{\text {age }}=15.10\right.$ years, $\mathrm{SD}_{\text {age }}=1.63$ years; 822 boys, 908 girls, eight did not specify their sex). For analysis purposes, the sample was divided into two age groups: 935 adolescents aged $12-15$ years $\left(\mathrm{M}_{\text {age }}=13.86\right.$ years, $\mathrm{SD}_{\text {age }}=1.07$ years; 454 boys, 480 girls, one nonspecified $)$ and 795 aged $16-18$ years $\left(\mathrm{M}_{\text {age }}=16.56\right.$ years, $\mathrm{SD}_{\text {age }}=0.67$ years; 367 boys, 427 girls, one non- specified). Eight participants who did not provide their ages were not included in the age-based categorization.

\section{Measures}

ERQ-CA-J: The ERQ-CA-J used in Study 2 was the same scale as that developed in Study 1.

Behavior for Regulating Emotions Scale: The Behavior for Regulating Emotions $\mathrm{Scale}^{24}$ is an 11-item measure of behavior regarding regulating emotions. The scale has 
a three-factor structure: positive reappraisal (four items; e.g., "to calm negative emotions, I try to think of the event as a good experience"), distraction (three items; e.g., "to calm negative emotions, I engage in recreation"), and emotional expression (four items; e.g., "to feel positive emotions, I do something to release my emotions"). Each item is rated using a six-point scale ( $1=$ "strongly disagree" to $6=$ "strongly agree").

Trait Subscale of State-Trait Anxiety Inventory: The Trait subscale of State-Trait Anxiety Inventory (STAI) ${ }^{25,26}$ is a 40-item measure of anxiety symptoms. The STAI has a two-factor structure: a state anxiety subscale and trait anxiety subscale. In the present research, participants rated the trait subscale (T-STAI), which comprises 20-items, with each item rated using a four-point scale $(1=$ "almost never" to 4 = "almost always").

Center for Epidemiologic Studies Depression Scale: The Center for Epidemiologic Studies Depression Scale $(\mathrm{CES}-\mathrm{D})^{27,28}$ is a 20 -item measure of depressive symptoms. The CES-D has a single-factor structure and each item is rated using a four-point scale $(0=$ "not at all" to 3 = "over five days a week").

\section{Statistical Analyses}

The software program and estimator used for analysis, criterion of model fit, and the Pearson's correlations were the same as that in Study 1. For construct validity, regarding the correlation between ES and other validity measures, we hypothesized that it would show no relationship with positive reappraisal, a weak positive relationship with distraction, a weak negative relationship with emotional expression, a weak positive relationship with anxiety, and a weak positive relationship with depression. Further, for $\mathrm{CR}$, it was hypothesized that it would show a moderate positive relationship with positive reappraisal, a moderate positive relationship with distraction, a moderate positive correlation with emotional expression, a moderate negative relationship with anxiety, and a moderate negative relationship with depression.

\section{Results and Discussion \\ Descriptive Statistics}

The means and standard deviations for the two emotion regulation strategies (ES and CR) are shown in Table 1. The means and standard deviations were almost the same as those of the original version. ${ }^{8}$

\section{Structural Validity}

We conducted EFA on subsample 1, to examine the unique factorial structure for the Japanese sample. The visual scree test indicated two-factor structure same as the original version as most appropriate. Table 2 shows the rotated factor pattern matrix and extraction communalities. Two factors explained $52.44 \%$ of the variance of ERQ-CA scores. Factor loadings on the first factor (CR) ranged $0.47-0.75$ and the second factor (ES), 0.52-0.78. The two factors were correlated $(\mathrm{r}=0.43)$. Thus, the EFA indicated the Japanese version of the ERQ-CA has a two-factor structure same as the original version.

We conducted CFA on subsample 2, to confirm the results provided by EFA. Using CFA, a two-factor model, based on the findings of Gross and John, ${ }^{7}$ showed acceptable fit $(\mathrm{CFI}=0.901$, RMSEA $=0.086)$. Following the recommendations of Gullone and Taffe, ${ }^{8}$ a correlated error was added between item 1 ("when I want to feel happier, I think about something else") and item 3 ("when I want to feel better (e.g., less sad, angry, or worried), I think about something else"), which have similarly worded phrases, and this produced a better fit $(\mathrm{CFI}=0.923, \mathrm{RMSEA}=0.077$, see Table 3 ). In this model, both groups aged $12-15$ years $(\mathrm{CFI}=$ 0.930$, RMSEA $=0.075)$ and $16-18$ years $(\mathrm{CFI}=0.910$, RMSEA $=0.080)$ showed acceptable model fit. Item-factor loadings of CR ranged from 0.44 to 0.73 (0.41-0.73 for ages 12-15 years; $0.45-0.71$ for ages $16-18$ years), and ES ranged from 0.50 to $0.74(0.51-0.73$ for ages $12-15$ years, $0.48-0.74$ for ages 16-18 years, see Table 4), which was considered sufficient. Therefore, the soundness of the ERQ-CA-J's twofactor structure was the same as that of Gullone and Taffe. ${ }^{8}$

\section{Internal Consistency Reliability}

Cronbach's alpha was 0.73 for the ES items (0.71 for boys, 0.75 for girls, 0.73 for age $12-15$ years, and 0.71 for age $16-18$ years), and was 0.79 for CR items ( 0.80 for boys, 0.76 for girls, 0.79 for ages $12-15$ years, and 0.77 for ages 16-18 years). Thus, the ERQ-CA-J was internally consistent.

\section{Construct Validity}

We conducted correlation analyses for two ERQ-CA-J factors with each validity scales: the subscales of Behavior for Regulating Emotions Scale (positive reappraisal, distraction, and emotional expression), T-STAI, and CES-D (Table 6). The correlations between the ES score and other validity scales were as expected, and the 
correlations between the CR score and behaviors for regulating emotions subscales were also as expected.

However, the associations between the CR score and some measures for validity investigation differed from expectations. There was almost no correlation between the T-STAI and CR scores and CES-D. This may be because the $\mathrm{CR}$ items were misinterpreted as a form of avoidance; it is possible that the participants mistakenly believed that the $\mathrm{CR}$ items did not concern changing their ways of thinking but related to thinking about something completely different.

\section{Study 3}

The purpose of Study 3 was to investigate the measurement invariance across the children and adolescent age groups and the scale's test-retest reliability over four weeks.

\section{Methods}

\section{Participants and Procedures}

The institutional review board (IRB) at the University of Tsukuba approved this study's ethical and scientific protocols (approval number: 筑29-117). Participants were recruited from two elementary schools, three middle schools, and two high schools in Japan. The procedure was the same as in Study 1 for elementary school children and the same as in Study 2 for middle school and high school adolescents.
Overall, 1329 participants completed a second questionnaire to assess test-retest reliability for the ERQ-CA-J with a four-week gap between the two surveys, which was based on Hasani's ${ }^{29}$ study of the ERQ's test-retest reliability over a five-week period. Of these participants, 29 were omitted because they had significant missing data (leaving $>20 \%$ of the items blank for at least one measure). This resulted in a sample of 1300 participants $\left(\mathrm{M}_{\text {age }}\right.$ $=13.53$ years, $\mathrm{SD}_{\text {age }}=2.48$ years; 656 boys, 644 girls). In the CFA, the data for the first period were used. For analysis purposes, the sample was divided into three age groups, 265 children aged $8-11$ years $\left(M_{\text {age }}=9.75\right.$ years, $\mathrm{SD}_{\text {age }}=0.98$ years; 134 boys, 131 girls), 736 adolescents aged 12-15 years $\left(\mathrm{M}_{\mathrm{age}}=13.60\right.$ years, $\mathrm{SD}_{\mathrm{age}}=1.05$ years; 389 boys, 347 girls), and 299 adolescents aged 16-18 years $\left(\mathrm{M}_{\mathrm{age}}=16.70\right.$ years, $\mathrm{SD}_{\mathrm{age}}=0.70$ years; 133 boys, 166 girls). Participants were volunteers and received no reward for their participation.

\section{Measures}

ERQ-CA-J: The ERQ-CA-J used in Study 3 was the same scale as that developed in Study 1 and used in Study 2. The participants completed the measure twice, four weeks apart.

\section{Statistical Analyses}

The software program and estimator used for analysis and criterion of model fit were the same as those in Study 1.

Table 6 Study 2 Correlations

\begin{tabular}{|c|c|c|c|c|c|c|}
\hline & & \multicolumn{3}{|c|}{ Behavior for Regulating Emotions Scale } & \multirow[t]{2}{*}{ T-STAI } & \multirow[t]{2}{*}{ CES-D } \\
\hline & & Positive Reappraisal & Distraction & Emotional Expression & & \\
\hline \multirow[t]{2}{*}{ Overall Sample } & ES & $0.08 * *$ & $0.13 * *$ & $-0.11 * *$ & $0.24 * *$ & $0.22 * *$ \\
\hline & CR & $0.46 * *$ & $0.49 * *$ & $0.36 * *$ & 0.00 & $-0.08 * *$ \\
\hline \multirow[t]{2}{*}{ Girls } & ES & 0.00 & 0.06 & $-0.21^{* *}$ & $0.25^{* *}$ & $0.24 * *$ \\
\hline & CR & $0.44 * *$ & $0.48^{* *}$ & $0.32 * *$ & $-0.08^{*}$ & $-0.16 * *$ \\
\hline \multirow[t]{2}{*}{ Boys } & ES & $0.19 * *$ & $0.22 * *$ & 0.02 & $0.24^{* *}$ & $0.20 * *$ \\
\hline & CR & $0.48 * *$ & $0.50 * *$ & $0.39 * *$ & $0.09 *$ & 0.01 \\
\hline \multirow[t]{2}{*}{ Age $12-15$ years } & ES & $0.12^{* *}$ & $0.16 * *$ & $-0.09 *$ & $0.27^{* *}$ & $0.23 * *$ \\
\hline & CR & $0.46 * *$ & $0.53^{* *}$ & $0.38 * *$ & 0.01 & -0.07 \\
\hline \multirow[t]{2}{*}{ Age $16-18$ years } & ES & 0.02 & 0.07 & $-0.15^{* *}$ & $0.20 * *$ & $0.20 * *$ \\
\hline & CR & $0.47^{* *}$ & $0.43 * *$ & $0.33 * *$ & -0.05 & $-0.12 * *$ \\
\hline
\end{tabular}

Notes: $*_{p}<0.05,{ }^{*} p<0.01$.

Abbreviations: CES-D, Center for Epidemiologic Studies Depression Scale; CR, cognitive reappraisal; ES, expressive suppression; T-STAI, Trait subscale of State-Trait Anxiety Inventory. 


\section{Results and Discussion \\ Descriptive Statistics}

The means and standard deviations for the two emotion regulation strategies (ES and CR) are shown in Table 1 . These means and standard deviations were almost the same as those of the original version. ${ }^{8}$

\section{Confirming Structural Validity}

Through CFA, the same model as that used in Study 1 and 2 showed acceptable fit (CFI $=0.908$, RMSEA $=0.075$, see Table 3). In this model, the fitness was calculated for each age group (8-11 years $(\mathrm{CFI}=0.897$, RMSEA $=$ $0.079), 12-15$ years $(\mathrm{CFI}=0.876$, RMSEA $=0.086)$, and $16-18$ years $(\mathrm{CFI}=0.912$, RMSEA $=0.074)$. Although the CFIs of the $8-11$ years and $12-15$ years groups were slightly lower than the criteria recommended by $\mathrm{Hu}$ and Bentler, ${ }^{22}$ RMSEA showed acceptable fit; thus, the model can be considered to have acceptable fit. Item-factor loadings of CR ranged from 0.45 to 0.69 ( $0.46-0.68$ for ages $8-11$ years, $0.41-0.69$ for ages $12-15$ years, and $0.45-0.72$ for ages 16-18 years), and ES ranged from 0.48 to 0.77 (0.45-0.69 for $8-11$ years, $0.44-0.78$ for $12-15$ years, and $0.49-0.88$ for ages $16-18$ years; see Table 4 ), which were considered to be sufficient. Therefore, the soundness of the ERQ-CA-J's two-factor structure was the same as that of Gullone and Taffe. ${ }^{8}$

\section{Test of Measurement Invariance}

As a further investigation, we tested measurement invariance, determining whether the ERQ-CA-J was invariant among the participants' age groups. Measurement invariance was tested on three distinct levels: (a) configural invariance (i.e., whether similar factors are measured in each age group), (b) factor-loading invariance (i.e., whether the magnitude of factor loadings is the same across each age group), and (c) intercept invariance (i.e., whether the intercept of the regression relating each item to its factor is the same). $\mathrm{Chen}^{30}$ recommended exploring practical model fit changes: if $\Delta \mathrm{CFI} \leq 0.010, \Delta \mathrm{RMSEA} \leq 0.015$ for factor-loading invariance, then measurement invariance is evidenced. The results of the investigation of measurement invariance are shown in Table 3. Although the configural invariance model had poor fit with regard to CFI (CFI $=0.889)$, the fit based on the RMSEA was good $($ RMSEA $=0.047)$. The factor-loading invariance model also showed poor fit for CFI $(\mathrm{CFI}=0.890)$, good fit for RMSEA (RMSEA $=0.044)$. The intercept invariance model showed a poor fit for CFI $(\mathrm{CFI}=0.863)$ and good fit for RMSEA (RMSEA $=0.045$ ). The model fit changes between the configural invariance model and factorloading invariance model $(\Delta \mathrm{CFI}=0.001, \Delta \mathrm{RMSEA}=0.003)$ met the criterion by Chen. ${ }^{30}$ The model fit changes between the factor-loading invariance model and intercept invariance model met the criterion for RMSEA ( $\triangle$ RMSEA $=0.001)$, but not for CFI $(\triangle \mathrm{CFI}=0.027)$. Therefore, the measurement invariance between age groups was not clearly demonstrated.

\section{Test-Retest Reliability}

The intraclass correlation coefficients (ICCs) were used to estimate the stability of the score of the ERQ-CA-J over the four weeks. The ICCs should be 0.75 or higher to show adequate time stability. ${ }^{31}$ For the CR subscale, the ICCs were 0.76 ( 0.74 for ages $8-11$ years, 0.75 for ages $12-15$ years, and 0.79 for ages $16-18$ years); for the ES subscale, the ICCs were 0.76 ( 0.73 for ages $8-11$ years, 0.76 for ages $12-15$ years, and 0.75 for ages $16-18$ years). These findings indicate the ERQ-CA-J had adequate test-retest reliability over the four-week period.

\section{General Discussion}

In the present research, we developed and investigated the psychometric evaluation of the ERQ-CA-J using three samples. Overall, the ERQ-CA-J's factor structure was the same as the original version, and internal consistency, test-retest reliability, and construct validity were supported for those aged 8-18 years.

It is noteworthy that there were some results that differed from expectations; in both Study 1 and 2, the correlations between the $\mathrm{CR}$ scores and some other validity scales were unexpected. In Study 1, CR and ES showed almost the same correlation patterns between other validity measures; this may be because CR and ES were not clearly differentiated by the children. Children, and to some extent adolescents, do not always have the neurobiological or cognitive capacities to regulate their emotions. ${ }^{32}$ As they get older, thinning of lateral prefrontal cortical areas during development facilitates improvement of adolescents' cognitive emotion regulation abilities. ${ }^{33}$ In addition, it can be said for both Study 1 and 2 that the CR items could easily have been misunderstood as relating to forms of avoidance. It is possible that the participants mistakenly understood the CR items as not relating to changing their ways of thinking, but to thinking about something completely different. For example, when a child is anxious that he/she may be hated by a peer, the child can, using CR strategies, think of another possibility, such as "the peer does not necessarily hate me." On the other hand, the child 
can also think about something completely different, such as "what is for dinner today?"-which constitutes a form of avoidance.

In Study 3, the measurement invariance between age groups was not clearly demonstrated. This finding could be because emotion regulation develops during childhood and adolescence, with better regulation and therefore higher intercepts as they grow older. The ability to utilize cognitively complex processes (e.g., cognitive reappraisal) increases through childhood to adolescence, along with the capacity to flexibly use a wider range of strategies in response to emotions. ${ }^{34,35}$

This study had some limitations. First, only self-report measures were used in the present study, relying on individuals' accuracy and honesty. In future, observational or semi-structured interview instruments could be used to eliminate response bias. Second, the internal consistency, test-retest reliability, factor structure, and validity of the ERQ-CA were all determined using classic psychometrics (e.g., using correlation analyses). An emerging model, the clinimetric approach, ${ }^{36}$ an innovative, clinically based measurement method, has recently been introduced to evaluate not only the psychometric properties but also the clinical validity of rating scales. While classic psychometrics mainly focuses on assessing the frequency of psychometric properties, using self-rating scales where all items have the same weight, clinimetric analysis allows for the treatment of syndromes as heterogeneous constructs with potential differences in weight. ${ }^{37}$ In future studies, the clinimetric approach may serve as an innovative method for examining the clinical validity of the ERQ-CA. Third, our sample did not contain diverse cultures, and future research should include children and adolescents from diverse cultural backgrounds to enable intercultural comparison, especially in relation to emotion regulation differences between Western and Eastern participants. ${ }^{11}$ Such a project would clarify the causal factors of differences found between the original and Japanese versions in the current study.

With the present research's development of the ERQCA-J, further studies concerning emotion regulation can be conducted in Japan. Emotion dysregulation is considered both a risk and maintenance factor for psychiatric disorders, particularly emotional disorders such as those relating to depression and anxiety. ${ }^{38}$ It is desirable to conduct psycho-education regarding emotion regulation for not only clinical populations, but also non-clinical children and adolescents as a means of preventing psychopathology, such as depression and anxiety.

\section{Acknowledgments}

We thank Dr. Eleonora Gullone, the developer of the original questionnaire, for granting us permission to develop the ERQ-CA-J. Part of this manuscript was presented as an abstract at the International Conference on Adolescent Medicine \& Child Psychology.

\section{Disclosure}

This research was supported by a grant from the Kawano Masanori Memorial Public Interest Incorporated Foundation for the Promotion of Pediatrics. Although the corresponding author is Hikari Namatame, the copyright holder of the ERQCA-J is Hiroko Fujisato. Hikari Namatame reports grants from Kawano Masanori Memorial Public Interest Incorporated Foundation for the Promotion of Pediatrics, during the conduct of the study. Hiroko Fujisato reports grants from Kawano Masanori Memorial Public Interest Incorporated Foundation for Promotion of Pediatrics, during the conduct of the study. Yoko Sawamiya reports grants from Kawano Masanori Memorial Public Interest Incorporated Foundation for the Promotion of Pediatrics, during the conduct of the study. Masaya Ito reports grants from Kawano Masanori Memorial Public Interest Incorporated Foundation for Promotion of Pediatrics, during the conduct of the study. The authors report no other conflicts of interest in this work.

\section{References}

1. Compas BE, Jaser SS, Bettis AH, et al. Coping, emotion regulation, and psychopathology in childhood and adolescence: a meta-analysis and narrative review. Psychol Bull. 2017;143:939. doi:10.1037/ bul0000110

2. Fabes RA, Eisenberg N, Miller PA. Maternal correlates of children's vicarious emotional responsiveness. Dev Psychol. 1990;26:639. doi:10.1037/0012-1649.26.4.639

3. Silk JS, Steinberg L, Morris AS. Adolescents' emotion regulation in daily life: links to depressive symptoms and problem behavior. Child Dev. 2003;74:1869-1880. doi:10.1046/j.1467-8624.2003.00643.x

4. Gross JJ. Antecedent-and response-focused emotion regulation: divergent consequences for experience, expression, and physiology. J Pers Soc Psychol. 1998;74:224-237. doi:10.1037/0022-3514.74.1.224

5. Gross JJ. Emotion and emotion regulation. In: Pervin LA, John OP, editors. Handbook of Personality: Theory and Research. 2nd ed. New York: Guildford Press; 1999:525-552.

6. Gross JJ. Emotion regulation in adulthood: timing is everything. Curr Dir Psychol Sci. 2001;10:214-219. doi:10.1111/1467-8721.00152

7. Gross JJ, John OP. Individual differences in two emotion regulation processes: implications for affect, relationships, and well-being. J Pers Soc Psychol. 2003;85:348-362. doi:10.1037/0022-3514.85.2.348 
8. Gullone E, Taffe J. The Emotion Regulation Questionnaire for Children and Adolescents (ERQ-CA): a psychometric evaluation. Psychol Assess. 2012;24:409-417. doi:10.1037/a0025777

9. Pepping CA, Duvenage M, Cronin TJ, Lyons A. Adolescent mindfulness and psychopathology: the role of emotion regulation. J Individ Differ. 2016;99:302-307. doi:10.1016/j.paid.2016.04.089

10. Yoshizu J, Sekiguchi R, Amemiya T.Development of a Japanese version of the emotion regulation questionnaire. Jpn J Res on Emot.2013;20:56-62. (in Japanese). doi:10.4092/jsre.20.56.

11. Butler EA, Lee TL, Gross JJ. Emotion regulation and culture: are the social consequences of emotion suppression culture-specific? Emot. 2007;7:30-48. doi:10.1037/1528-3542.7.1.30

12. Mokkink LB, Terwee CB, Patrick DL, et al. COSMIN checklist manual. Available from: http://www.cosmin.nl/images/upload/files/ COSMIN\%20checklist\%20manual\%20v9.pdf.

13. Sato Y, Fujita M, Uno H. Developing the Children's emotional regulation scale. J Child Health. 2013;72:525-530. (in Japanese).

14. Spence SH. A measure of anxiety symptoms among children. Behav Res Ther. 1998;36:545-566. doi:10.1016/S0005-7967(98)00034-5

15. Ishikawa SI, Sato H, Sasagawa S. Anxiety disorder symptoms in Japanese children and adolescents. $J$ Anxiety Disord. 2009;23:104-111. doi:10.1016/j.janxdis.2008.04.003

16. Birleson P. The validity of depressive disorder in childhood and the development of a self-rating scale: a research report. J Child Psychol Psychiatry. 1981;22:73-88. doi:10.1111/jcpp.1981.22.issue-1

17. Murata T, Shimizu A, Mori Y, Oshima S. Childhood depressive states in school situations: considerations using Birleson's scale. Jpn J Psychiatry. 1996;1:131-138. (in Japanese).

18. Birleson P, Hudson I, Buchanan DG, Wolff S. Clinical evaluation of a self-rating scale for depressive disorder in childhood (depression self-rating scale). $J$ Child Psychol Psychiatry. 1987;28:43-60. doi:10.1111/jcpp.1987.28.issue-1

19. Cattell RB. The scree test for the number of factors. Multivar Behav Res. 1966;1:245-276. doi:10.1207/s15327906mbr0102 10

20. Floyd FJ, Widaman KF. Factor analysis in the development and refinement of clinical assessment instruments. Psychol Assess. 1995;7:286. doi:10.1037/1040-3590.7.3.286

21. Bentler PM, Chu CP. Practical issues in structural modelling. Sociol Meth Res. 1987;16:78-117. doi:10.1177/0049124187016001004

22. Hu LT, Bentler PM. Cutoff criteria for fit indexes in covariance structure analysis: conventional criteria versus new alternatives. Struct Equ Modeling. 1999;6:1-55. doi:10.1080/10705519909540118

23. Cohen J. Statistical Power Analysis for the Behavioral Sciences. 2nd ed. Hilsdale. NJ: Lawrence Earlbaum Associates; 1988.
24. Nozaki Y. Effects of regulations of the self and others on changes in emotional intelligence during a term examination period. Jpn J Educ Psychol.2013;61:362-373. (in Japanese). doi:10.5926/jjep.61.362.

25. Spielberger CD. STAI manual for the state-trait anxiety inventory. Self-Evaluation Questionnaire. 1970:1-24.

26. Nakazato K, Mizuguchi T. Development and validation of a Japanese version of the state-trait anxiety inventory: a study with female subjects. Shinshin-Igaku. 1982;22:107-112. (in Japanese).

27. Radloff LS. The CES-D scale: a self-report depression scale for research in the general population. App Psychol Meas. 1977;1:385-401. doi:10.1177/014662167700100306

28. Shima S, Shikano T, Kitamura T, Asai M. A new self-report depression scale. Psychiatry. 1985;27:717-723. (in Japanese).

29. Hasani J. Persian version of the emotion regulation questionnaire: factor structure, reliability and validity. Int $J$ Behav Sci. 2017;10:156-161.

30. Chen FF. Sensitivity of goodness of fit indexes to lack of measurement invariance. Struct Equ Modeling. 2007;14:464-504. doi:10.1080/10705510701301834

31. Shrout PE, Fleiss JL. Intraclass correlations: uses in assessing rater reliability. Psychol Bull. 1979;86:420-428. doi:10.1037/00332909.86.2.420

32. Steinberg L, Dahl R, Keating D, Kupfer DJ, Masten AS, Pine DS. The study of developmental psychopathology in adolescence: integrating affective neuroscience with the study of context. In: Cicchetti D, Cohen DJ. editors. Developmental Psychopathology: Developmental Neuroscience. Vol. 2, 2nd ed. Hoboken, NJ: Wiley;2006:710-741.

33. Vijayakumar N, Whittle S, Yücel M, Dennison M, Simmons J, \& Allen NB. Thinning of the lateral prefrontal cortex during adolescence predicts emotion regulation in females. Soc Cog Affect Neuros. 2014;9:1845-1854.

34. Skinner EA, Zimmer-Gembeck MJ. The development of coping. Annu Rev Psychol. 2007;58:119-144.

35. Zimmer-Gembeck MJ, Skinner EA. The development of coping across childhood and adolescence: An integrative review and critique of research. Int J Behav Dev. 2011;35:1-17.

36. Bech P. Clinimetric dilemmas in outcome scales for mental disorders. Psychoth Psychos. 2016;85:323-326.

37. Fava GA, Ruini C, Rafanelli C. Psychometric theory is an obstacle to the progress of clinical research. Psychoth Psychos. 2004;73:145-148.

38. Barlow DH, Allen LB, Choate ML. Toward a unified treatment for emotional disorders-republished article. Behav Ther. 2016;47:838853 .
Neuropsychiatric Disease and Treatment

\section{Publish your work in this journal}

Neuropsychiatric Disease and Treatment is an international, peerreviewed journal of clinical therapeutics and pharmacology focusing on concise rapid reporting of clinical or pre-clinical studies on a range of neuropsychiatric and neurological disorders. This journal is indexed on PubMed Central, the 'PsycINFO' database and CAS, an is the official journal of The International Neuropsychiatric Association (INA). The manuscript management system is completely online and includes a very quick and fair peer-review system, which is all easy to use. Visit http://www.dovepress.com/testimonials.php to read real quotes from published authors. 